\title{
Downregulation of cylindromatosis gene, $C Y L D$, confers a growth advantage on malignant melanoma cells while negatively regulating their migration activity
}

\author{
YUICHI ISHIKAWA ${ }^{1-3}$, KANAKO TSUNODA ${ }^{3}$, MASAHIKO SHIBAZAKI ${ }^{1}$, KAZUHIRO TAKAHASHI ${ }^{3}$, \\ TOSHIHIDE AKASAKA ${ }^{3}$, TOMOYUKI MASUDA ${ }^{2}$ and CHIHAYA MAESAWA ${ }^{1}$ \\ ${ }^{1}$ Department of Tumor Biology, Institute of Biomedical Science, Departments of ${ }^{2}$ Pathology \\ and ${ }^{3}$ Dermatology, School of Medicine, Iwate Medical University, Iwate, Japan
}

Received December 26, 2011; Accepted February 5, 2012

DOI: 10.3892/ijo.2012.1424

\begin{abstract}
The cylindromatosis gene (CYLD) encodes a deubiquitinase that was initially identified as a tumor suppressor and has recently been investigated in connection with a variety of normal physiological processes. In contrast to its cell-proliferative activity, the effect of CYLD protein on cell migration has been a matter of debate. We investigated the effect of $C Y L D$-siRNA on the migration activity of malignant melanoma cells. Expression of $C Y L D$ mRNA/protein was lower in 6 of 8 malignant melanoma cell lines than in 3 sets of primarycultured normal human epidermal melanocytes. Knockdown of $C Y L D$ significantly increased the proliferation activities of two melanoma cell lines $(\mathrm{p}<0.05)$, along with BCL3 nuclear translocation followed by $C C N D 1$ overexpression. In contrast to the proliferation-related activity, $C Y L D$ knockdown significantly decreased the cell migration of all the melanoma cell lines $(n=7$, $\mathrm{p}<0.05$ ), and we demonstrated that the mechanism regulating melanoma cell migration was activation of RAC1 through the action of $C Y L D$. Our findings provide new insight into the role of $C Y L D$-induced RACl activation in melanoma cell migration.
\end{abstract}

\section{Introduction}

CYLD (cylindromatosis) was originally identified as a tumor suppressor gene that is mutated in familial cylindromatosis (Brooke-Spiegler syndrome), an autosomal-dominant predisposition to multiple tumors of skin appendages (1). The CYLD gene encodes a deubiquitinating enzyme, which removes lysine 48- or lysine 63-linked polyubiquitin chains from target proteins (2). Depending on the cellular context, $C Y L D$ has been shown to negatively regulate the NF- $\mathrm{kB}$ and/or JNK-signaling pathways resulting in suppression of cell proliferation and survival (3-6).

Correspondence to: Dr Chihaya Maesawa, Department of Tumor Biology, Institute of Biomedical Science, Iwate Medical University, 2-1-1 Nishitokuta, Yahaba-cho, Iwate 028-3694, Japan

E-mail: chihaya@iwate-med.ac.jp

Key words: melanoma, CYLD, migration, proliferation, HDAC6
The mechanism by which $C Y L D$ exerts its tumor-suppressor function in vivo has been analyzed in $C Y L D$-null mice, which are highly susceptible to chemically-induced skin tumors. This increased tumor incidence has been attributed to loss of an inhibitory interaction between CYLD and the proto-oncogene BCL3. The association of CYLD with BCL3, which results from activation and subsequent perinuclear translocation of the protein, leads to the removal of a lysine 63-linked ubiquitin chain from BCL3, which in turn inhibits the nuclear translocation and activity of BCL3 (7). In the absence of CYLD gene expression, BCL3 is able to translocate into the nucleus where it forms a complex with the NF- $\mathrm{NB}$ p50 and p52 isoforms. This results in activation of the cyclin Dl (CCNDI) promoter and increased proliferation and tumor growth (7).

In contrast to the proliferation activity of tumor cells, the effect of $C Y L D$ protein in cell migration has been a matter of debate. Some $C Y L D$ mutants mimicking mutations that have been identified in human cylindromatosis contribute to the acquisition of metastatic potential by tumor cells (8-10). However, Gao et al $(11,12)$ have demonstrated that $C Y L D$ downregulation causes a decrease of cell migration activity in both cancer and endothelial cells. The $C Y L D$ gene is known to contain 3 cytoskeleton-associated protein glycine-rich (CAP-Gly) domains, which exist in a number of microtubule-binding proteins and are responsible for their association with microtubules (MT). Knockdown of cellular CYLD expression dramatically delays MT regrowth after nocodazole washout, indicating a role for CYLD in promoting MT assembly (11). Recently, direct association between $C Y L D$ and histone deacetylase-6 (HDAC6), a cytoplasmic histone deacetylase, has been documented (13). The stability of MT is accompanied by post-translational modifications of $\alpha$-tubulin (TUBA1) such as acetylation, which usually occurs after MT assembly (14). The level of TUBA1 acetylation in cells is accurately adjusted by the activities of tubulin acetyltransferases and deacetylases, which catalyze the acetylation and deacetylation of TUBA1, respectively. ELP3 was identified very recently as TUBA1 acetyltransferase in neuronal cells (15), while HDAC6 has been shown to deacetylate TUBA1 (16). Even though HDAC6 contains both intrinsic nuclear import and export signals, it is found mainly in the cytoplasm (17), where it localizes around the nucleus or at the leading edge of migrating 
cells (16). Loss of HDAC6 function is involved in cell migration activity through several molecular mechanisms $(18,19)$.

HDAC6 is involved in multiple cellular processes, such as cell migration and adhesion, endocytosis, and stress responses. Overexpression of HDAC6 protein has been identified in a variety of cancer cells and mouse tumor models. The levels of HDAC6 expression in ovarian cancer cells and tissues were reportedly higher in low-grade and high-grade ovarian carcinomas than in benign lesions and immortalized ovarian surface epithelial cell lines (20). HDAC6 expression was also upregulated in primary oral squamous cell lines and its level of expression was correlated with primary tumor stage (21). Upregulation of HDAC6 in a variety of tumors and cell lines suggests an important role in cancer, and it is therefore being widely investigated.

We also recently demonstrated that the deacetylation system of nucleus accumbens-associated 1 (NACC1) and HDAC6 can modulate the status of both TUBA1 and cortactin (CTTN), which is an actin-crosslinking protein (22). Motility of melanoma cells was affected via MT- and actin-dependent processes through the NACC1/HDAC6 deacetylation system. Moreover, overexpression of HDAC6 is a good prognostic and metastatic biomarker in human melanomas (22). The aggressiveness of malignant melanoma is characterized by high metastatic ability and resistance to chemotherapy and immunotherapy (23-26). If $C Y L D$ negatively regulates HDAC6 function, it might act as a repressor of metastasis. In the present study, we examined the expression of the CYLD gene in malignant melanoma cell lines and primary cultures of normal human epidermal melanocytes (NHEMs), and investigated its association with tumor cell migration and proliferation activities.

\section{Materials and methods}

Cell culture. Five human melanoma cell lines (HMV-I, HMV-II, SK-MEL-28, CRL1579, and G-361) were obtained from the Cell Resource Center for Biomedical Research, Tohoku University (Sendai, Japan). Three melanoma cell lines (PM-WK, MM-AN, and MM-RU) were donated by Professor M.C. Mihm (Department of Dermatology, Harvard Medical School, Boston, MA, USA). They were maintained at $37^{\circ} \mathrm{C}$ under $5 \% \mathrm{CO}_{2}$ in RPMI-1640 (Invitrogen, Carlsbad, CA, USA) supplemented with $10 \%$ fetal bovine serum (Invitrogen). Three primary cultures of NHEMs (NHEM-D, NHEM-L, and NHEM-M) were purchased from Kurabo Co. (Osaka, Japan) and maintained at $37^{\circ} \mathrm{C}$ under $5 \% \mathrm{CO}_{2}$ in Medium 254 (Invitrogen) with the addition of human melanocyte growth supplement (Invitrogen) and gentamicin/ amphotericin solution (Invitrogen).

Antibodies. For immunofluorescence, polyclonal anti-CYLD antibody (Cell Signaling Technology, Danvers, MA, USA) and polyclonal anti-BCL3 (H-146) (Santa Cruz Biotechnology, Santa Cruz, CA, USA) were used. 4'-6-diamino-2-phenylindole (DAPI) (Wako Pure Chemical Industries, Ltd., Osaka, Japan) solution was used to stain nuclei. The secondary antibody was Alexa Fluor $^{\circledR} 488$ anti-rabbit IgG (Invitrogen). For immunoblotting of CYLD and BCL3, we used the same primary antibody as that used for immunofluoresence. The other primary antibodies were monoclonal CCND1 (cyclin D1) (Cell Signaling Technology), polyclonal LMNB1 (lamin B) (Santa Cruz Biotechnology), monoclonal TUBA1 ( $\alpha$-tubulin) (Sigma Chemical Co. Ltd., St. Louis, MO, USA), and monoclonal anti-GAPDH, Clone 1D4 (Covance, Princeton, NJ, USA).

Western blotting. All cell lines were cultured to $70-80 \%$ confluence on 10-cm Petri dishes. Cold PBS (phosphate-buffered saline) was added, and the cells were removed from the dishes by scraping. After removal of the supernatants, the cell pellet was dissolved in cell lysis buffer [50 mM Tris- $\mathrm{HCl}, \mathrm{pH} 8.0 / 150 \mathrm{mM}$ $\mathrm{NaCl} / 1$ mM EDTA, pH 8.0/1\% Triton $\mathrm{X}-100 / 0.1 \%$ sodium deoxycholate/0.1\% SDS/1 mM PMSF/10 mM NaF/2 mM $\mathrm{Na}_{3} \mathrm{VO}_{4} / 1 \mathrm{X}$ complete protease inhibitor (Roche Diagnostics $\mathrm{GmbH}$, Mannheim, Germany)]. Cell samples containing equal amounts of protein were mixed with $5 \mathrm{X}$ sample buffer, and heated for $5 \mathrm{~min}$ at $95^{\circ} \mathrm{C}$. Protein was electrophoresed on $4-12 \%$ $\mathrm{Nu}-\mathrm{PAGE}$ for $45 \mathrm{~min}$ at $200 \mathrm{~V}$ constant voltage, and then transferred onto polyvinylidene difluoride membranes (Invitrogen) for $1 \mathrm{~h}$ at $30 \mathrm{~V}$ constant voltage. The membranes were blocked with $5 \%$ blocking reagent (Cell Signaling Technology) in $1 \mathrm{X}$ Tris-buffered saline/Tween-20 buffer for $1 \mathrm{~h}$ at room temperature, and immunostained with primary antibodies overnight at $4^{\circ} \mathrm{C}$. Then, the membranes were rinsed with Tris-buffered saline/ Tween-20 and incubated with horseradish HRP-conjugated secondary antibodies [anti-rabbit or -mouse IgG (GE Healthcare, Little Chalfont, UK)] for $1 \mathrm{~h}$ at room temperature. Signals were detected with ECL Prime (GE Healthcare) and ChemiDoc XRS (Bio-Rad Laboratories, Hercules, CA, USA). The intensity of the detected signals was measured using 1-D analysis software (Quantity One, Bio-Rad). For normalization of the target, GAPDH, LMNB1 or TUBA1 was used as an internal control.

Separation of nuclear and cytoplasmic fractions. Nuclear and cytoplasmic extracts from cells were prepared using a NE-PER Nuclear and Cytoplasmic Extraction Reagent Kit (Thermo Scientific, Rockford, IL, USA), in accordance with the manufacturer's protocol. Briefly, the cells were pelleted by centrifugation at $500 \mathrm{x} g$ for $3 \mathrm{~min}$ and the supernatants discarded. To obtain cytoplasmic extracts, ice-cold CERI (Thermo Scientific) buffer was added to the pellets. After vortexing and $10 \mathrm{~min}$ of incubation on ice, ice-cold CERII (Thermo Scientific) buffer was added for further incubation. Further centrifugation was conducted for $5 \mathrm{~min}$, and the supernatants (cytoplasmic extracts) were immediately transferred to new tubes and placed on ice. The insoluble pellets were suspended in ice-cold NER buffer (Thermo Scientific). After vortexing and incubating on ice, the tubes were centrifuged at $16,000 \mathrm{x} \mathrm{g}$ for $10 \mathrm{~min}$. The supernatants (nuclear fractions) were immediately transferred to clean, prechilled tubes. Extracts were stored at $-80^{\circ} \mathrm{C}$ until Western blotting experiments.

RNA isolation and quantitative reverse transcriptase PCR. Total RNA was extracted with TRIzol reagent (Invitrogen), and transcribed to cDNA using a SuperScript III First-Strand Synthesis System(Invitrogen). For quantitative evaluation of the relevant mRNAs, we used Custom TaqMan Gene Expression Assays (CYLD, Hs00211000_m1; CCND1, Hs00765553_ml; Invitrogen), and an ABI PRISM 7500 instrument (Invitrogen). For normalization of the target, glyceraldehyde-3-phosphate dehydrogenase (GAPDH, Invitrogen) was used as an internal control. All reactions (each containing 3 templates) were run 
in triplicate, and average fold differences were calculated by normalizing the relative expression ( $\Delta \Delta \mathrm{Ct}$ values) according to ABI User Bulletin no. 2.

siRNA knockdown of the CYLD gene. For silencing of CYLD gene mRNA, 3 predesigned $C Y L D$-specific siRNA sequences (no. 1, s590; no. 2, s591; no. 3, s592; Silencer Select Predesigned siRNA, Invitrogen), and control non-specific human siRNAs (Silencer Select Predesigned siRNA Negative Control no. 1, 4390843; no. 2, 4390844, Invitrogen) were used. siRNA transfection was performed using Lipofectamine RNAiMAX Reagent (Invitrogen) according to the manufacturer's instructions.

Confocal microscopy. Cells were washed with PBS and fixed with $4.0 \%$ formaldehyde and $0.5 \%$ Triton X-100 for $20 \mathrm{~min}$. The slides were then blocked with $5 \%$ bovine serum albumin for $30 \mathrm{~min}$ at room temperature. After 3 washes with PBS, the slides were incubated with a primary antibody in blocking buffer at $4^{\circ} \mathrm{C}$. The slides were incubated with a secondary antibody for $3 \mathrm{~h}$ at $37^{\circ} \mathrm{C}$. Cells were counterstained with DAPI and imaged with a Nikon EZ-C1 confocal microscope (Nikon Co., Tokyo, Japan).

RACl activity assay. An Active Rac1 Pull-Down and Detection Kit was purchased from Thermo Scientific and experiments were performed in accordance with the manufacturer's protocol. Lysed samples in RIPA buffer were incubated with $20 \mathrm{ml}$ of glutathione S-transferase-human Pak1-p21-binding domain for $1 \mathrm{~h}$ at $4^{\circ} \mathrm{C}$, followed by 3 times with wash buffer. Proteins bound to agarose beads were eluted in $50 \mathrm{ml}$ of $2 \mathrm{X}$ SDS sample buffer and subjected to Western blot analysis.

Tumor cell migration assay. Confluent cell monolayers were scratched to create a wound, and then 0,24 and $48 \mathrm{~h}$ later, 3 different fields of each wound were photographed with a phase-contrast microscope. Three independent experiments were performed. Measurements of the width of each wound were performed under each experimental condition. At the start of the experiment, the wound size was measured and scored as $100 \%$. After 24 and $48 \mathrm{~h}$, the width of the residual wound was measured and the average percentage of wound closure was calculated by using the free web software package Image $\mathrm{J}$ (http://rsb.info.nih. gov/ij).

Cell proliferation assay.Cell proliferation assays were performed using a Cell Counting Kit-8 (Dojindo Co., Kumamoto, Japan). Cells were plated in 96-well plates at $1 \times 10^{4}$ cells per well and cultured in the growth medium. Cell numbers in triplicate wells were measured as the absorbance $(450 \mathrm{~nm})$ of reduced WST-8 [2-(2-methoxy-4-nitrophenyl)-3-(4-nitrophenyl)-5-(2,4disulfophenyl)-2H-tetrazolium, monosodium salt].

Statistical analysis. All data are presented as means \pm standard error. Correlations between CYLD protein expression and clinicopathological data were analyzed by Fisher's exact test. To correlate the results of CYLD expression with overall survival in patients with malignant melanomas, Kaplan-Meier survival analysis and log-rank test were performed. Mann-Whitney U test for non-parametric samples was used for biological experimental data analyses. The level of significance was considered to be $\mathrm{p}<0.05$.
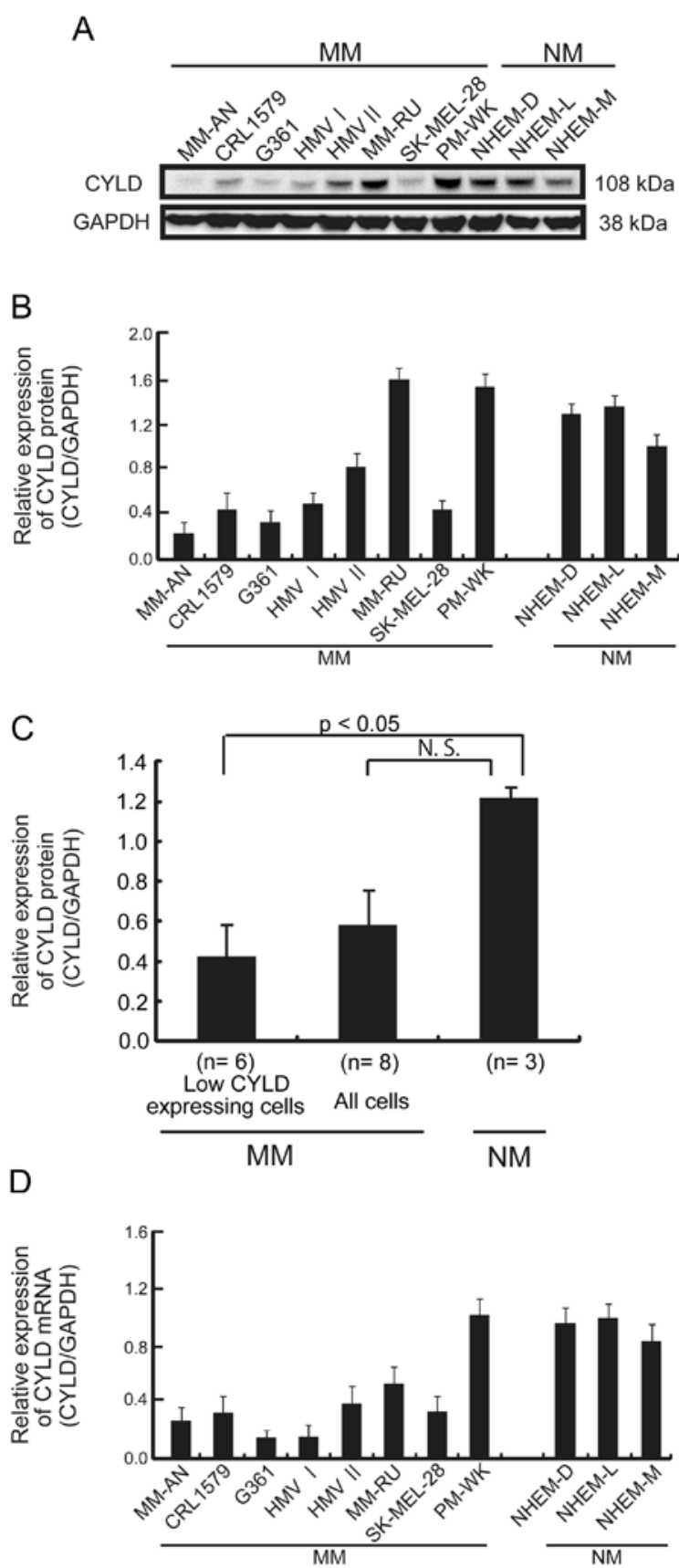

Figure 1. Expression of $C Y L D$ protein/mRNA in 8 melanoma cell lines and 3 primary cultures of normal human epidermal melanocytes (NHEMs). (A) Western blotting for CYLD protein in 8 melanoma cell lines and 3 primary cultures of NHEMs. Equal loading was confirmed by blotting GAPDH. (B and C) Quantitative results of Western blotting for CYLD protein in 8 melanoma cell lines and 3 primary cultures of NHEMs. (C) Six of the 8 melanoma cell lines exhibited relatively lower CYLD protein expression in comparison with primary cultures of NHEMs. (D) Results of quantitative RT-PCR analysis for CYLD were compatible with protein expression. GAPDH, glyceraldehyde-3-phosphate dehydrogenase. MM, malignant melanoma. NM, normal melanocyte. NS, not significant.

\section{Results}

Expression of the CYLD gene in cell lines and primary tumors of malignant melanomas. We first examined mRNA/protein expression of $C Y L D$ in 8 melanoma cell lines and 3 primary cultures of NHEMs (Fig. 1). Under the recommended condi- 


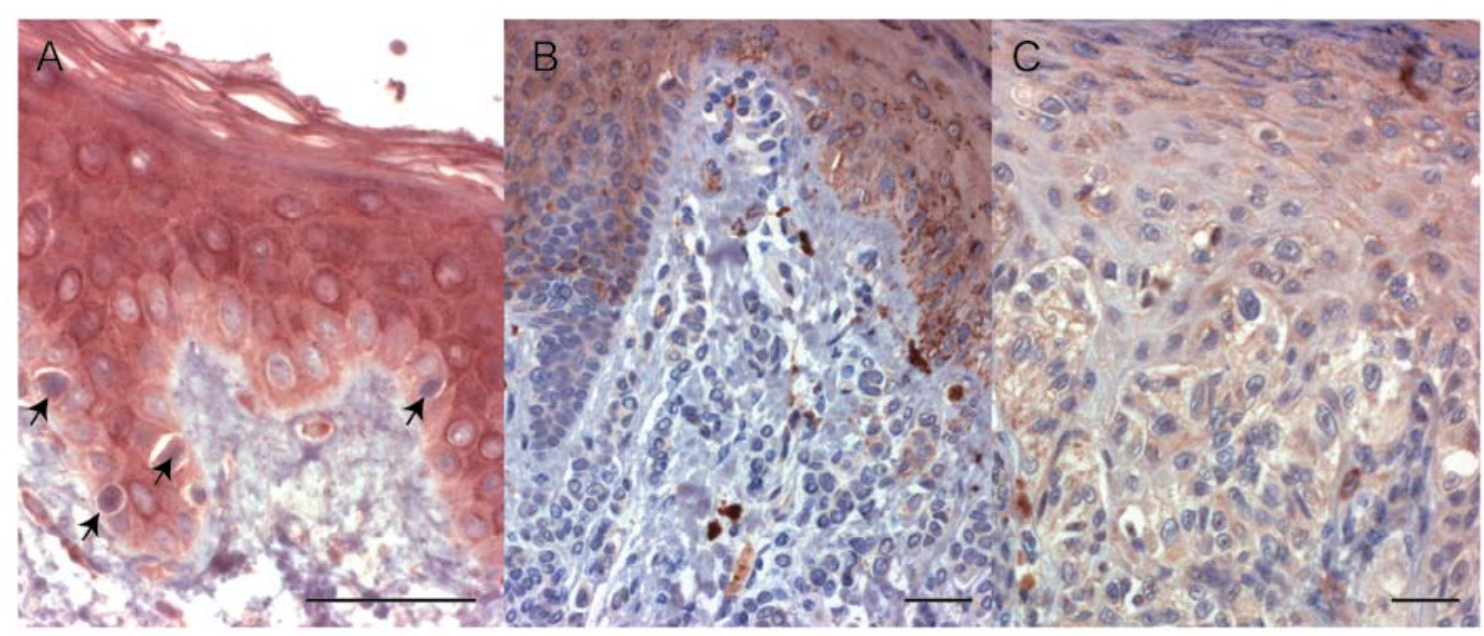

Figure 2. Immunohistochemistry for CYLD protein in human normal skin and primary malignant melanomas. (A) CYLD immunoreactivity in normal skin. Positive signals were observed in epidermal keratinocytes, but the basal layer showed relatively weak expression. CYLD protein was positive in both the cytoplasm and nucleus of epidermal melanocytes (arrows). (B) Negative immunostaining for CYLD in tumor cells of a malignant melanoma. (C) Weakly positive signals in tumor cells of a malignant melanoma. Scale bars, $100 \mu \mathrm{m}$.

tions and at $60-70 \%$ confluency, expression of CYLD protein decreased in $6(75 \%)$ of the 8 melanoma cell lines in comparison with 3 NHEMs (Fig. 1). Two melanoma cell lines (MM-RU and PM-WK) expressed CYLD protein at levels similar to those in the 3 NHEMs (Fig. 1A-C). Expression of CYLD mRNA was consistent with that of the protein (Fig. 1D).

Next, we evaluated CYLD protein expression in 49 patients with malignant melanomas and in 10 with non-neoplastic skin disease. Dense immunoreactivity for CYLD protein was observed in both skin keratinocytes and epidermal melanocytes (Fig. 2A). Immunoreactivity for CYLD protein was negative in 40 (82\%) of 49 primary melanomas (Fig. 2B), and the remaining $9(18 \%)$ exhibited a weak reaction (Fig. 2C). Although we investigated the relationships between CYLD immunoreactivity and clinicopathological data for the 49 melanoma patients and their disease outcome, no significant relationship was evident.

Cell migration activity induced by treatment with CYLD-siRNA in melanoma cell lines. We first evaluated the knockdown efficiency of CYLD-siRNAs (nos. 1, 2 and 3; $10 \mathrm{nM}$ ) in a malignant melanoma cell line (PM-WK, Fig. 3). In comparison with negative control siRNA, all the siRNAs caused $80-90 \%$ downregulation of both $C Y L D$ mRNA and protein expression (Fig. 3). Using no. 1 CYLD siRNA, we then examined phenotypic changes in the migration activities of the 7 melanoma cell lines (except for HMV-I, as its growth pattern was unsuitable for the scratch assay). $C Y L D$ knockdown significantly decreased the migration activity of all 7 melanoma cell lines in comparison with the negative control at 24 and $48 \mathrm{~h}(\mathrm{p}<0.05$, Mann-Whitney U test; Fig. 4).

Effects of treatment with CYLD-siRNA on microtubule acetylation in melanoma cell lines. We evaluated MT after CYLD knockdown by siRNA treatment in two $C Y L D$-overexpressing melanoma cell lines (MM-RU and PM-WK). Accumulation of acetylated tubulin around the nucleus was decreased in comparison with control siRNA treatment in both cell lines (Fig. 5A), whereas no marked change of total TUBA1 distribution was
A
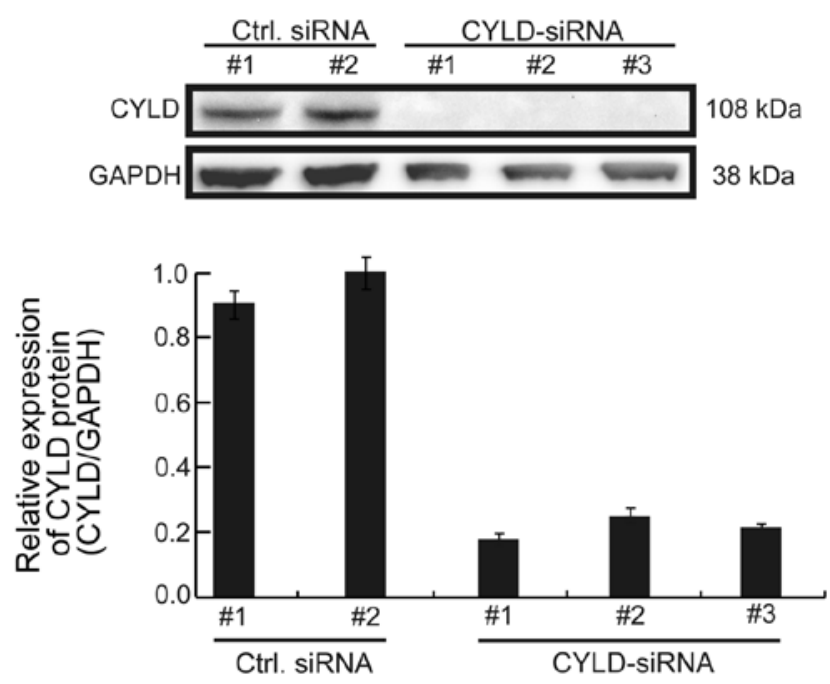

B

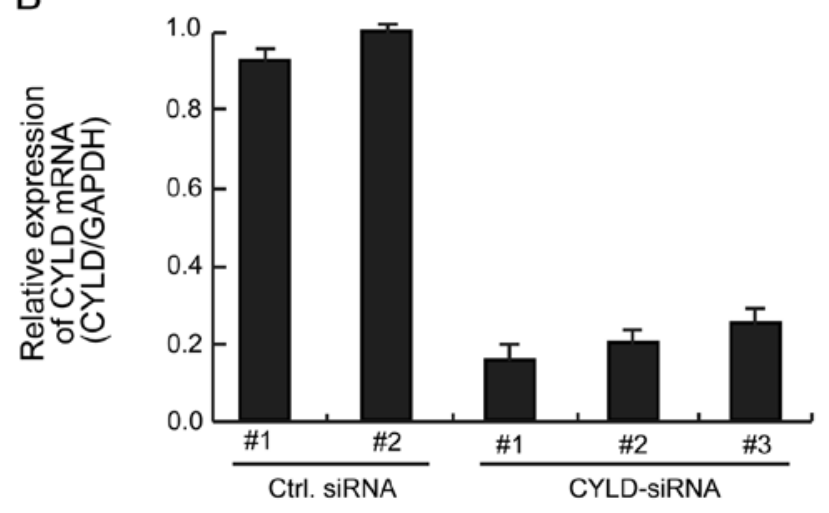

Figure 3. Knockdown efficiency of $C Y L D$-siRNA used in the present study. Expression of both $C Y L D$ protein (A) and mRNA (B) was decreased by $>80 \%$.

found throughout the cytoplasm. Immunoblot analyses showed that $C Y L D$ knockdown induced a decrease of acetylated tubulin in comparison with control siRNA (Fig. 5B). Knockdown of 
A

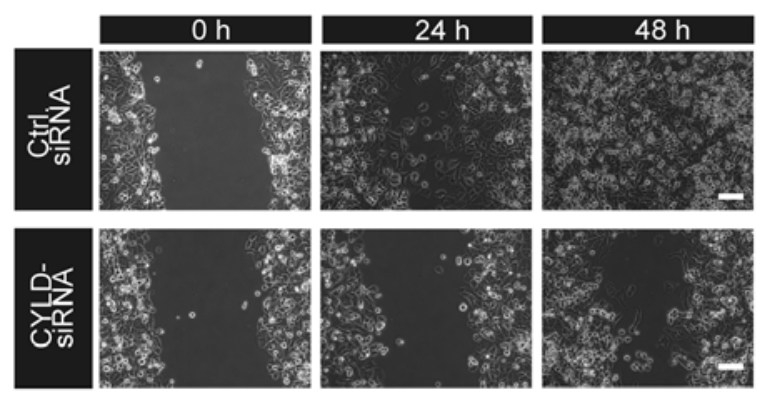

B

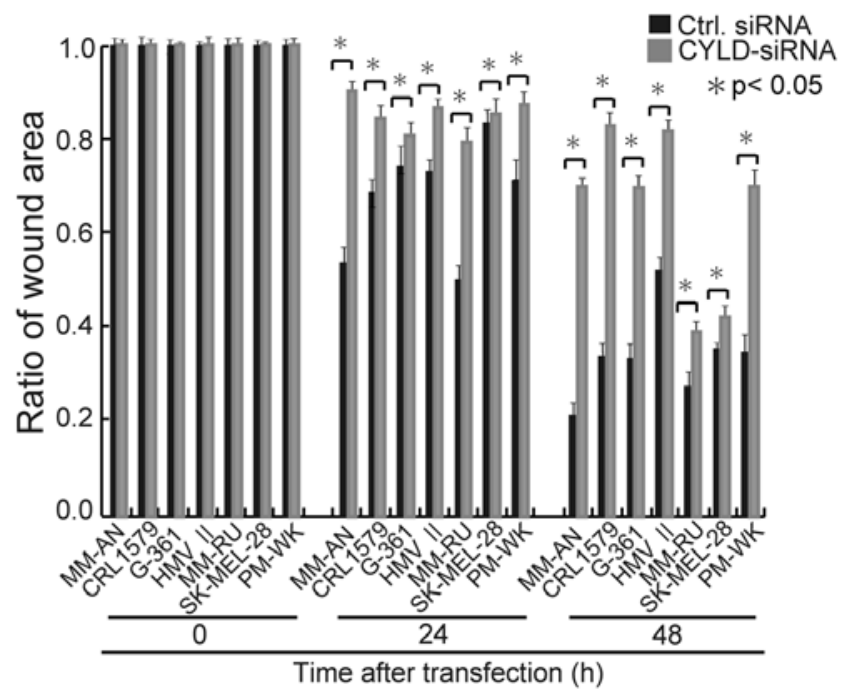

Figure 4. Migration activity induced by treatment with $C Y L D$-siRNA in melanoma cell lines. (A) Photographs of scratch assays for MM-WK (scale bars, $100 \mu$ m) and (B) quantification of seven melanoma cell lines at 24 and $48 \mathrm{~h}$ after transfection with control (ctrl.) or CYLD-siRNA (10 nM). Cell migration activity (inversely correlated with the wound area ratio) was significantly decreased in cells treated with CYLD-siRNA at 24 and $48 \mathrm{~h}$.

A

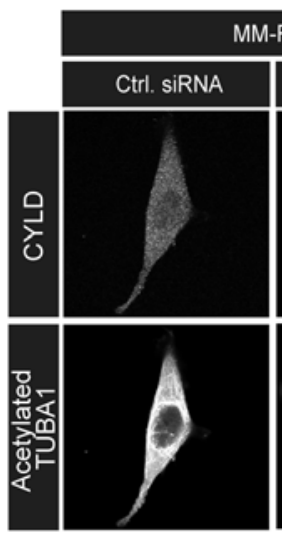

\section{M-RU}
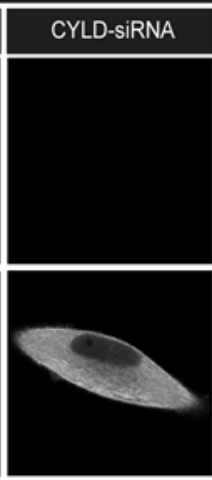

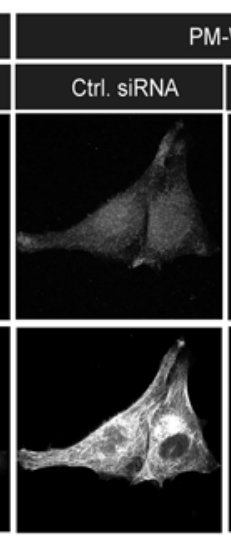

B

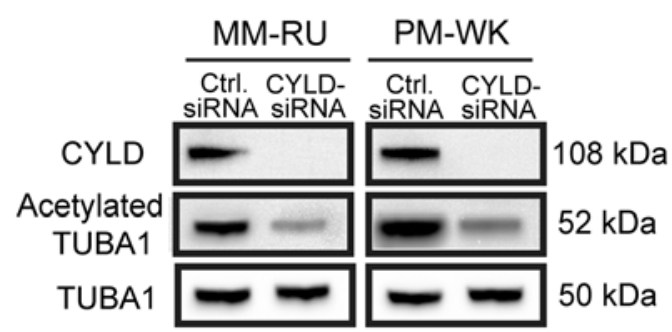

Figure 5. Acetylation status of microtubules after treatment with CYLD-siRNA. (A) Confocal microscopy observations of CYLD and acetylated TUBA1 in MM-RU and PM-WK cells treated with siRNAs for CYLD, and (B) immunoblotting for CYLD and acetylated TUBA1. Bundles of acetylated tubulin was decreased around the nucleus in cells treated with $C Y L D$-siRNA in comparison with control (ctrl.) siRNA. Acetylated TUBA1 was markedly decreased by CYLD knockdown in both cell lines (B).

CYLD gene induced the activation of HDAC6 deacetylation, and might introduce dynamic state of MT.

Activation of RACl by treatment with CYLD-siRNA in melanoma cell lines. A contradiction was found between MT acetylation status and migration activity in two melanoma cell lines treated with $C Y L D$-siRNA. Next, we tested RAC1 activation in these cells, because it has recently been clarified that silencing of $C Y L D$ is involved in MT dynamics and represses the migration activity of endothelial cells, suggesting that repression of active RAC1 contributes to a decrease of endothelial cell migration (12). We also confirmed a marked decrease of active RACl expression ( $\mathrm{p}<0.05)$ in both melanoma cell lines (Fig. 6), in accord with the results of Gao et al (12).
Effects of treatment with CYLD-siRNA on proliferation activity of melanoma cell lines. Treatment with $C Y L D$-siRNA decreased the proliferation of two $C Y L D$-overexpressing melanoma cell lines (Fig. 7A). We also identified BCL3 translocation to the nucleus by confocal microscopy (Fig. 7B) and Western blot analyses using nuclear and cytoplasmic extracts (Fig. 7C), as described previously (7). This in turn induced overexpression of CCNDl mRNA/protein (Fig. 8).

\section{Discussion}

At the start of this study, we had hypothesized that knockdown of the CYLD gene might accelerate the motility of melanoma cells, because $C Y L D$ inhibits HDAC6 deacetylation activity 
A

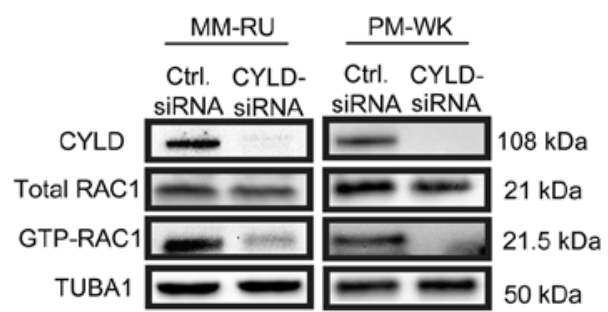

B
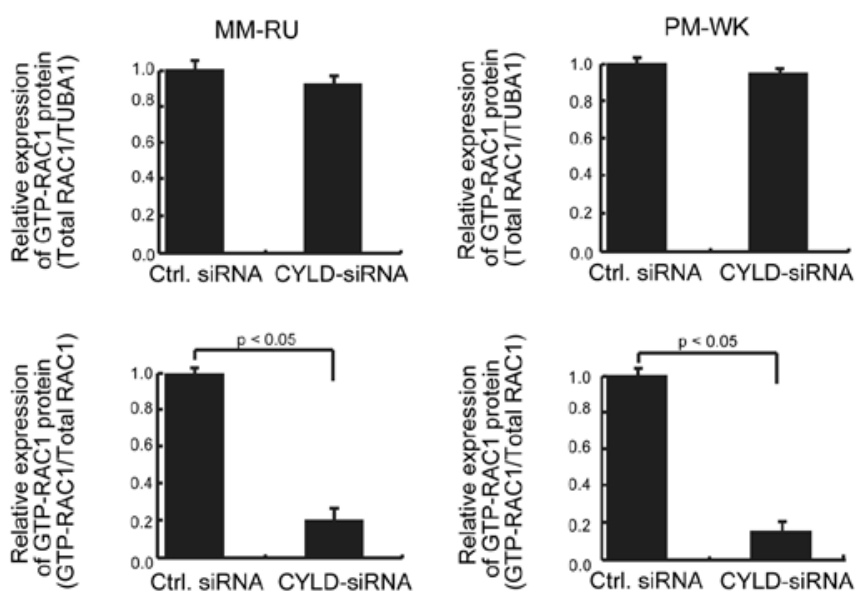

Figure 6. Effects of $C Y L D$ knockdown on expression of GTP-RAC1. Active RAC1 pull-down and immunoblotting (A), and its quantification (B). MM-RU and PM-WK cells were transfected with control (ctrl.) or CYLD-siRNA $(10 \mathrm{nM})$. In both cell lines, expression of GTP-RAC1 was significantly decreased by CYLD knockdown.

(13). We recently reported that disruption of HDAC6 was closely associated with a decease of in vitro migration and invasion activities of melanoma cells (22). Inactivation of HDAC6 induced hyperacetylation of not only TUBA1 (13) but also CTTN (22). It introduced stabilization of MT dynamicity, and prevented the translocation of cortactin protein to the cell periphery, thus blocking its association with F-actin, and impairing the motility of melanoma cells. Moreover, $C Y L D$ downregulation is associated with aggressive tumor behavior in the development of human cancers including not only malignant melanoma but also tumors of the colon, lung and kidney, and also cervical and hepatocellular carcinomas $(10,27)$. Considering these in vitro and in vivo findings as a whole, our hypothesis pertaining to the relationship between $C Y L D$ and the motility of melanoma cells would appear to be reasonable. However, our experiments yielded findings that were contradictory to this concept.

Massoumi and colleagues (10) demonstrated that mitogenic and metastatic properties of melanoma cells were promoted by downregulation of $C Y L D$ through the action of Snaill, whose expression is induced by $B R A F$-mediated activation of extracellular signal-regulated kinase $(E R K)$. Snaill-dependent inhibition of $C Y L D$ transcription results in stimulation of cyclin DI and $N$-cadherin expression in melanoma cells, leading to enhanced proliferation, migration, and invasiveness of melanoma cells in vitro, as well as tumor growth and metastasis in vivo (10). In addition, tumors developing in $C Y L D$-truncated mutant mice were reportedly prone to malignant progression and lymph node metastasis along with activation of $c$-Jun-NH2-kinase (JNK) and the downstream c-Jun and c-Fos proteins (8). Moreover,
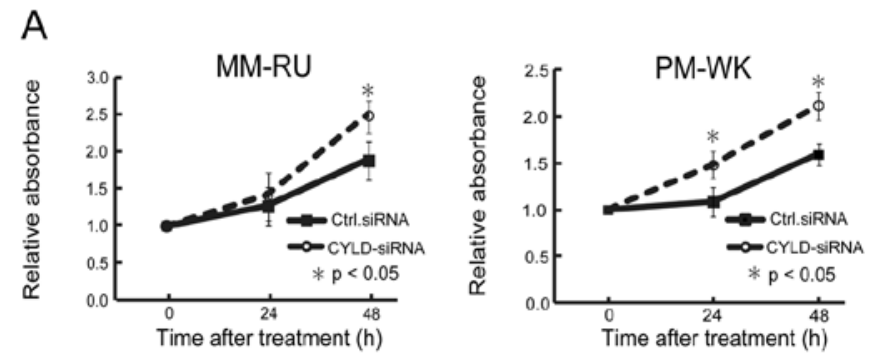

B

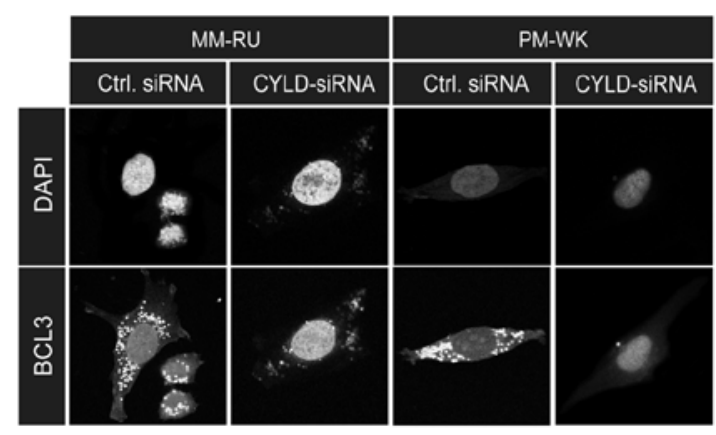

C
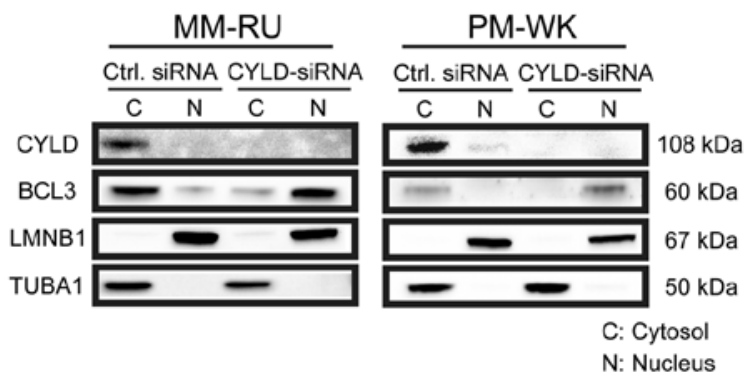

Figure 7. Proliferation activity and nuclear localization of BCL3 with CYLD knockdown. (A) Proliferation activity was increased by $C Y L D$ knockdown in both cell lines (MM-RU and PM-WK). (B) Confocal microscopy observations of BCL3 in MM-RU and PM-WK cells treated with control (ctrl.) or CYLD-siRNA at $48 \mathrm{~h}$. BCL3 was observed mainly in perinuclear areas after treatment with control siRNA, but in the nucleus after treatment with $C Y L D$ siRNA. (C) Immunoblotting for BCL3 protein. BCL3 was detected in the nuclear fraction of MM-RU and PM-WK after treatment with CYLD-siRNA. To ensure equal protein loading in the nuclear and cytosolic fractions, antiLMNB1 and anti-TUBA1 antibodies were used.

topical application of a pharmacologic JNK inhibitor significantly reduced tumor development and abolished metastasis in the transgenic mice (8).

Some reports have suggested that $C Y L D$ inactivation confers migration activity on tumor cells, contrary to the results obtained by Gao et al $(11,12)$. This discrepancy could be attributable to the method used for inactivation of $C Y L D$ functions. Massoumi et al $(2,10)$ and de Marval et al (8) used a murine model of patient-relevant mutations observed in familial cylindromatosis, these transgenic mice lacked the catalytic domain of the CYLD C-terminus and required TPA stimulation for development of skin tumors. In contrast to these studies, Gao et al employed simple $C Y L D$ knockdown by siRNA treatment, which repressed the cell migration activities of HeLa and endothelial cells, in accord with our results obtained using melanoma cell lines $(11,12)$. CYLD protein binds to both HDAC6 and TUBA1 through its N-terminal CAP-Gly domains, and increases the level of acetylated tubulin (13). The truncated C-terminus of CYLD protein might exert a dominant negative effect on deubiquitinase activity for the 
A

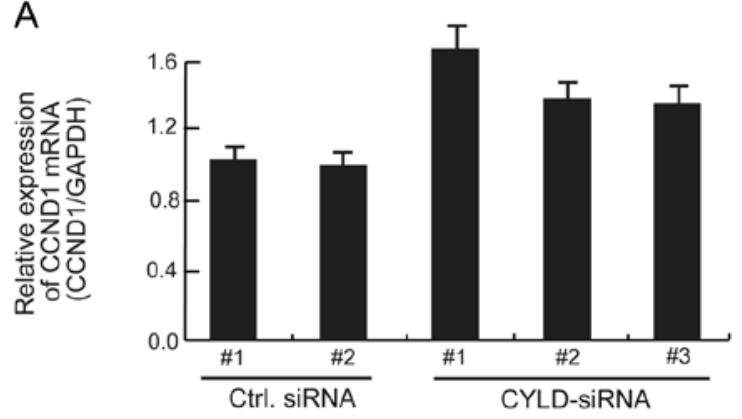

B
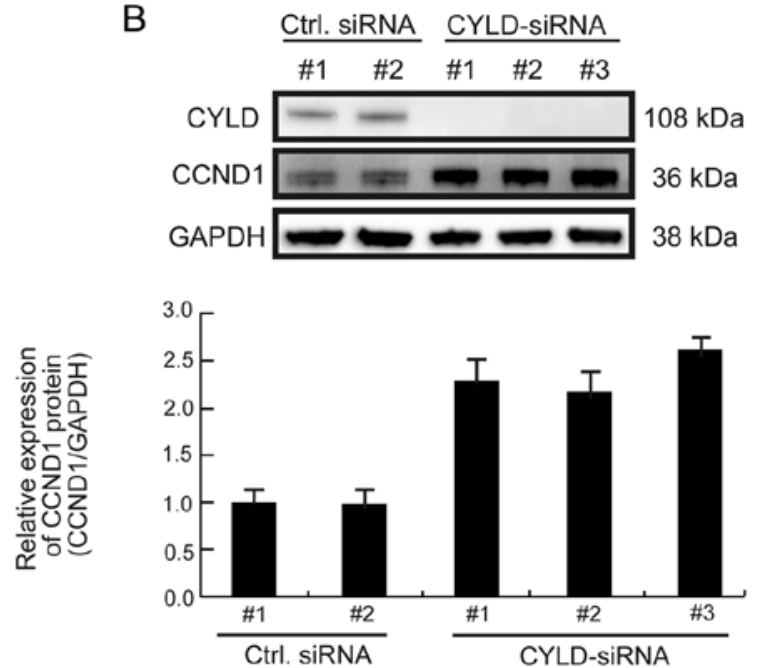

Figure 8. Immunoblotting for cyclin D1 (CCND1) after treatment with $C Y L D$ siRNA in a melanoma cell line (PM-WK). Expression of CCND1 mRNA (A) and protein (B) was increased in PM-WK treated with $C Y L D$-siRNA (48 h, $10 \mathrm{nM}$ ) in comparison with control (ctrl.) siRNA.

nuclear translocation of BCL3 and acquisition of cell proliferation. However, this would repress HDAC6 deacetylation activity, resulting in induction of MT hyperacetylation and stabilization of tumor cell motility. In fact, keratinocytes derived from Cyld null mice or melanoma cells treated with siRNA (presented in this study) showed activation of HDAC6 deacetylation activity and a decreased level of acetylated tubulin. However, the migration activity of these cells was markedly repressed. Taken together, these findings suggest that another molecular mechanism should be considered for the decrease of cell migration caused by knockdown of the CYLD gene.

Gao et al demonstrated that silencing of CYLD introduced RAC1 activation as an important factor contributing to the action of $C Y L D$ in regulating endothelial cell migration and angiogenesis (12), in accord with our results for melanoma cells. It is still unclear how $C Y L D$ is involved in RAC1 activation, and contributes to the effects of cell migration. One feasible idea is that $C Y L D$ may deubiquitinate RAC1 and thereby promote RAC1 activation to enable efficient cell migration. This notion is supported by observations that RAC1 activity is regulated by ubiquitination $(28,29)$. As $C Y L D$ is involved in melanoma cell migration, further studies of post-transcriptional regulation of MT and/or RAC1 could provide new targets for future therapeutic intervention to control melanoma cell motility.

\section{Acknowledgements}

This work was supported in part by Grants-in-Aid for Scientific Research (22390071, 23890198), and the MIAST project, and a Grant-in-Aid for the Strategic Medical Science Research Center from the Ministry of Education, Culture, Sports, Science and Technology of Japan.

\section{References}

1. Bignell GR, Warren W, Seal S, et al: Identification of the familial cylindromatosis tumour-suppressor gene. Nat Genet 25: 160-165, 2000.

2. Massoumi R and Paus R: Cylindromatosis and the CYLD gene: new lessons on the molecular principles of epithelial growth control. Bioessays 29: 1203-1214, 2007.

3. Brummelkamp TR, Nijman SM, Dirac AM and Bernards R: Loss of the cylindromatosis tumour suppressor inhibits apoptosis by activating NF-kappaB. Nature 424: 797-801, 2003.

4. Kovalenko A, Chable-Bessia C, Cantarella G, Israel A, Wallach D and Courtois G: The tumour suppressor CYLD negatively regulates NF-kappaB signalling by deubiquitination. Nature 424: 801-805, 2003.

5. Trompouki E, Hatzivassiliou E, Tsichritzis T, Farmer H, Ashworth A and Mosialos G: CYLD is a deubiquitinating enzyme that negatively regulates NF-kappaB activation by TNFR family members. Nature 424: 793-796, 2003.

6. Reiley W, Zhang M and Sun SC: Negative regulation of JNK signaling by the tumor suppressor CYLD. J Biol Chem 279: 55161-55167, 2004.

7. Massoumi R, Chmielarska K, Hennecke K, Pfeifer A and Fassler R: Cyld inhibits tumor cell proliferation by blocking Bcl-3-dependent NF-kappaB signaling. Cell 125: 665-677, 2006.

8. de Marval PM, Lutfeali S, Jin JY, Leshin B, Selim MA and Zhang JY: CYLD inhibits tumorigenesis and metastasis by blocking JNK/AP1 signaling at multiple levels. Cancer Prev Res 4: 851-859, 2011

9. Alameda JP, Moreno-Maldonado R, Navarro M, et al: An inactivating CYLD mutation promotes skin tumor progression by conferring enhanced proliferative, survival and angiogenic properties to epidermal cancer cells. Oncogene 29: 6522-6532, 2010.

10. Massoumi R, Kuphal S, Hellerbrand C, et al: Down-regulation of CYLD expression by Snail promotes tumor progression in malignant melanoma. J Exp Med 206: 221-232, 2009.

11. Gao J, Huo L, Sun X, et al: The tumor suppressor CYLD regulates microtubule dynamics and plays a role in cell migration. J Biol Chem 283: 8802-8809, 2008.

12. Gao J, Sun L, Huo L, Liu M, Li D and Zhou J: CYLD regulates angiogenesis by mediating vascular endothelial cell migration. Blood 115: 4130-4137, 2010.

13. Wickstrom SA, Masoumi KC, Khochbin S, Fassler R and Massoumi R: CYLD negatively regulates cell-cycle progression by inactivating HDAC6 and increasing the levels of acetylated tubulin. EMBO J 29: 131-144, 2010.

14. Westermann S and Weber K: Post-translational modifications regulate microtubule function. Nat Rev Mol Cell Biol 4: 938-947, 2003.

15. Creppe C, Malinouskaya L, Volvert ML, et al: Elongator controls the migration and differentiation of cortical neurons through acetylation of alpha-tubulin. Cell 136: 551-564, 2009.

16. Hubbert C, Guardiola A, Shao R, et al: HDAC6 is a microtubuleassociated deacetylase. Nature 417: 455-458, 2002.

17. Verdel A, Curtet S, Brocard MP, et al: Active maintenance of mHDA2/mHDAC6 histone-deacetylase in the cytoplasm. Curr Biol 10: 747-749, 2000.

18. Tran AD, Marmo TP, Salam AA, et al: HDAC6 deacetylation of tubulin modulates dynamics of cellular adhesions. J Cell Sci 120: 1469-1479, 2007.

19. Kwon S, Zhang Y and Matthias P: The deacetylase HDAC6 is a novel critical component of stress granules involved in the stress response. Genes Dev 21: 3381-3394, 2007.

20. Bazzaro M, Lin Z, Santillan A, et al: Ubiquitin proteasome system stress underlies synergistic killing of ovarian cancer cells by bortezomib and a novel HDAC6 inhibitor. Clin Cancer Res 14: 7340-7347, 2008. 
21. Sakuma T, Uzawa K, Onda T, et al: Aberrant expression of histone deacetylase 6 in oral squamous cell carcinoma. Int J Oncol 29: 117-124, 2006.

22. Tsunoda K, Oikawa H, Tada H, et al: Nucleus accumbens-associated 1 contributes to cortactin deacetylation and augments the migration of melanoma cells. J Invest Dermatol 131: 1710-1719, 2011.

23. Gajewski TF: Failure at the effector phase: immune barriers at the level of the melanoma tumor microenvironment. Clin Cancer Res 13: 5256-5261, 2007.

24. Postovit LM, Seftor EA, Seftor RE and Hendrix MJ: Influence of the microenvironment on melanoma cell fate determination and phenotype. Cancer Res 66: 7833-7836, 2006.

25. Satyamoorthy K and Herlyn M: Cellular and molecular biology of human melanoma. Cancer Biol Ther 1: 14-17, 2002.
26. Soengas MS and Lowe SW: Apoptosis and melanoma chemoresistance. Oncogene 22: 3138-3151, 2003.

27. Hellerbrand C, Bumes E, Bataille F, Dietmaier W, Massoumi R and Bosserhoff AK: Reduced expression of CYLD in human colon and hepatocellular carcinomas. Carcinogenesis 28: 21-27, 2007.

28. Visvikis O, Lores P, Boyer L, Chardin P, Lemichez E and Gacon G: Activated Racl, but not the tumorigenic variant Raclb, is ubiquitinated on Lys 147 through a JNK-regulated process. FEBS J 275: 386-396, 2008.

29. Kovacic HN, Irani K and Goldschmidt-Clermont PJ: Redox regulation of human Racl stability by the proteasome in human aortic endothelial cells. J Biol Chem 276: 45856-45861, 2001. 